\title{
Assistência ao parto em São Leopoldo (RS): um estudo de base populacional
}

\author{
Assistance to obstetrical deliveries in São Leopoldo, \\ Rio Grande do Sul State: a population-based study
}

M argareth Schreiner ${ }^{1}$

Juvenal Soares Dias da Costa ${ }^{2}$

Maria Teresa Anselmo Olinto ${ }^{2}$

Stela N azareth M eneghel ${ }^{2}$

${ }^{1}$ Departamento de Administração Hospitalar, Instituto Porto Alegreda I greja M etodista. RuaCel. Joaquim Pedro Salgado 80, Rio Branco. 90420-060 Porto Alegre RS. margareth.schreiner@ metodistadosul.edu.br 2 Programa de Pós-

Graduação em Saúde Coletiva, Universidade do Vale do Rio dos Sinos.
Abstract The study was carried out to describe the deliveries structural conditions, characteristics of assistance to the deliveries, to establish the percentage of cesarean section, and to verify the association between the presence of the pediatrician and the type of childbirth with socioeconomic variables. A cross-sectional populationbased study was carried out including 840 women from 20 to 49 years old resident in São Le opoldo, Rio Grande do Sul State. Among them, 36 (4.3\%) had children within 12 months prior the research. The percentage of women who had caesarian delivery was $52.8 \%$. Regarding the obstetric delivery, $97.2 \%$ had been assisted by a doctor and $72.2 \%$ of the women had reported the presence of pediatrician at the time of the labor. In $68.6 \%$ of the cases the delivery process was financed by the SUS, $20 \%$ were by private health care plans and $11.4 \%$ were privately paid. The caesarean delivery was less frequent among the women belonging to lower socioeconomic levels (prevalence rate $0.42 ; \mathrm{Cl} 195 \% 0.20-0.86$ ). It was verified that $30.4 \%$ of the women who had received maternal care by SUS did not report the presence of pediatrician in the labor. It was evidenced the association of the type of delivery and some variables related to economic class, making it possible to state that financial compensation intervenes with the type of obstetrical delivery. Key words O bstetric delivery, Cesarean section, Pediatric, Socioeconomics factors
Resumo 0 objetivo foi descrever as condições estruturais de assistência ao parto, estabelecer o percentual de cesarianas, as características deatenção ao parto e verificar a associação do tipo e presença de pediatra no parto com variáveis socioeconômicas. Foi realizado um estudo transversal de base populacional, incluindo 840 mulheres de 20 a 49 anos residentes na zona urbana de São Leopoldo (RS). Entre as 840 mulheres entrevistadas na pesquisa, $36(4,3 \%)$ tiveram filho nos doze meses anteriores à realização da pesquisa. 0 percentual de mulheres que tiveram parto cesáreo foi de $52,8 \%$. Quanto à realização do parto, $97,2 \%$ foram feitos por médico e $72,2 \%$ das mulheres re feriram a presença de pediatra no momento do parto. 0 financiamento do parto ocorreu em $68,6 \%$ doscasos pelo SUS, $20 \%$ foram financiados pelos planos privados de saúde $11,4 \%$ foram pagos deforma particular. 0 parto cesáreo foi menos frequente entre as mulheres de nível socioeconômico mais baixo ( $R P$ 0,42; I $C_{95 \%} 0,20-0,86$ ). Verificou-se que 30,4\% das mulheres que receberam cuidados pelo SUS não manifestaram presença de pediatra no momento do parto. Foram encontradas evidências mostrando a associação de tipo de parto e variáveis que expressam classe econômica, sendo possível afirmar que a remuneração interfere no tipo de parto.

Pal avras-chave Parto obstétrico, Cesárea, Pediatria, Fatores socioeconômicos 
Introdução

O Programa de Assistência Integral à Saúde da Mulher inclui entre seus diversos componentes a atenção ao parto, que está fortemente relacionada à mortalidade infantil ${ }^{1}$. Sabe-se, por exemplo, queem São Leopoldo eno Estado do Rio Grande do Sul as principais causas de morte entre os nascidos vivos são anóxia e hipóxia como representantes do grupo classificado como afecções originadas no período perinatal ${ }^{2}$. Este grupo de doenças pode estar relacionado com as condições de assistência oferecidas no momento do parto, tais como a presença ou não de pediatra para assistir ao recém-nascido.

Outro problema nacional relacionado à assistência ao parto é o elevado índice de cesarianas $^{3}$. De acordo com o estudo realizado na Amé rica Latina por Belizán et al. ${ }^{4}$, entre dezenovepaíses avaliados, sete deles apresentaram taxas de cesarianas abaixo de $15 \%$, sendo representados por Haiti, Guatemala, Bolívia, Peru, Paraguai, Honduras e El Salvador. No entanto, em doze países latino-americanos, as taxas variavam de $16,8 \%$ a $40 \%$, sendo que alguns apresentavam taxas elevadas, como o Chile (41,0\%), o Brasil $(27,1 \%)$, a República Dominicana $(25,9 \%)$ e a Argentina ( $25,4 \%)$, e outros com taxas menores, como Colômbia (16,8\%), Panamá (18,2\%) e Equador (18,5\%). A Organização Mundial da Saúde indica como parâmetros percentuais aceitáveis ao redor de 10\% para cesarianas. Sabe-se que, entre as complicações mais frequentes do parto tipo cesáreo, estão infecções puerperais, hemorragias, acidentes anestésicos eacidentes incisionais 5 .

Devido às elevadas taxas de cesarianas ocorridas no Brasil, o M inistério da Saúde implantou várias medidas na área de obstetrícia para incentivar o parto normal. Em maio de 1998, foi estabelecido que os procedimentos de partos normais poderiam ser feitos mediante anal gesia. Foi dado um reajuste geral do valor pago pelo parto e, além disso, foi criado o Sistema de Referência para Gestação de Alto Risco, constituído por maternidades que acompanham as parturientes com algum tipo de complicação (diabetes e hipertensão, por exemplo). Estes hospitais passaram a receber adicional de $25 \%$ ou $50 \%$ sobre os valores de tabela dos procedimentos realizados. Também foi incluído no sistema o parto realizado por enfermeiro obstetra. A variação do valor do parto normal, no período, foi de $162,6 \%$, passando de $R \$ 114,23$, em janeiro de 1995 , para $R \$$ $300,00 \mathrm{em} 2000^{3}$.
A instalação e aprimoramento do Sistema Único de Saúde (SUS) têm, ao longo dos anos, gerado a necessidade de incorporação do método epidemiológico para sua consolidação, conferindo racionalidadeao processo deplanejamento de sistemas e serviços de saúde. Assim, as informações coletadas podem subsidiar as etapas de diagnóstico de situação, a escolha de prioridades, a programação, a monitoração e a avaliação das atividades implementadas 6 .

Este estudo teve como objetivo descrever as condições estruturais de assistência ao parto, de estabelecer o percentual de cesarianas e de verificar a associação do tipo de parto e presença de presença de pediatra para assi stir o recém-nascido com variáveis socioeconômicas nas mulheres residentes em São Leopoldo, Rio Grande do Sul.

\section{M ateriais e métodos}

0 presente estudo é parte integrante do projeto "Condições de Saúde das Mulheres: Estudo de base populacional na Região do Vale do Rio dos Sinos - RS". Entre março e dezembro de 2003, foi realizado um estudo transversal de base populacional com o objetivo de caracterizar as condições de saúde das mulheres de vinte a sessenta anos, residentes na zona urbana de São Leopoldo (RS), através da investigação de múltiplos desfechos.

A cidade de São Leopoldo situa-se na região da encosta inferior do nordeste do Rio Grande do Sul, região do Vale do Rio dos Sinos. No último censo demográfico, tinha 193.547 habitantes, sendo que, deste total, 98.781 eram mulheres (51,07\%).

0 município conta na sua estrutura de serviços públicos de saúde com um hospital geral (Hospital Centenário) e 27 postos de saúde.

O projeto de pesquisa foi submetido eaprovado pelo Comitê de Ética e Pesquisa da Faculdade de M edicina da Universidade Federal de Pelotas.

Foram incluídas no presente estudo as muIheres de 20 a 49 anos que tiveram parto no último ano desde o momento da entrevista.

0 tamanho da amostra foi estimado a partir de diferentes desfechos previstos neste projeto, escolhendo-se aquele que necessitava de maior número de indivíduos. Calculou-se um tamanho de amostra que permitisse identificar uma razão de risco de 2,0, para um nível de confiança de $95,0 \%$, deum poder estatístico de $80,0 \%$, mantida a razão de não expostos:expostos de 1:3, segundo a estimativa inicial de distribuição de classe social. Portanto, optou-se por um tamanho 
de amostra que permitisse estudar o desfecho que exigisse maior tamanho de amostra: 1.086 pessoas. Considerando-se a necessidade de aumentar o tamanho da amostra em $10,0 \%$ devido a possíveis perdas e $15,0 \%$ para controlar fatores de confusão na análise dos dados, calculou-se como necessário um total de 1.358 pessoas. Desta forma, através de uma amostra por conglomerados, foram sorteados quarenta setores censitários entre os 270 existentes na zona urbana da cidade de São Leopoldo. A partir de um quarteirão previamentesorteado, escolheu-setambém de maneira al eatória o ponto de partida, saltando-se uma casa para selecionar o próximo domicílio. Foram visitados 36 domićlios, onde re sidiam pessoas do sexo feminino na faixa etária especificada. 0 número total de mulheres incluídas no estudo foi de 1.026, sendo que o número de perdas e recusas foi de 58 (5,65\%).

Foi aplicado um questionário padronizado e pré-codificado, por entrevistadores acadêmicos da UNISINOS, que receberam treinamento pré vio para aplicação dos questionários e padronização das medidas. 0 teste final dos instrumentos foi realizado através de estudo piloto e o controle dequalidadefoi realizado com a aplicação dequestionários simplificados em $10 \%$ da amostra.

Para descrição das variáveis de assistência ao parto, foi verificado o tipo de parto realizado (normal ou cesárea); local do parto (quanto ao hospital e quanto ao município de realização), financiamento do procedimento (SUS, planos privados de saúde ou médico particular), profissional que realizou o parto e presença de pediatra em sala de parto.

Entreas variáveis socioeconômicas, foi incluída a classificação econômica da Associação $\mathrm{Na}$ cional de Empresas de Pesquisa (ANEP) que levou em consideração a acumulação de bens materiais e anos de escolaridade do chefe da família. As outras variáveis socioeconômicas incluídas no estudo foram renda familiar per capita em salários mínimos e escolaridade da gestante.

A entrada dos dados foi realizada através do programa Epi Info, com dupla entrada e posterior checagem de inconsistências. As análises bruta e ajustada dos dados foram realizadas através do programa SPSS. Foram calculadas as razões de prevalências, os intervalos de confiança e os níveis de significância foram verificados através do teste exato de Fisher.

\section{Resultados}

Foram encontradas 1.026 mulheres de vinte a sessenta anos. Entre as 840 mulheres de 20 a 49 anos incluídas no estudo, $36(4,3 \%)$ relataram gravidez nos últimos doze meses antes do momento da entrevista. Destas, dezenove $(52,8 \%)$ tiveram parto cesáreo.

Quanto à assistência ao parto, foi constatado que sua totalidade foi em ambiente hospitalar, $97,2 \%$ foram realizados por médicose $71,4 \%$ ocorreram no município de São Leopoldo. As entrevistadas mencionaram presença de pediatra nasala de parto em $72,2 \%$ das ocasiões (Tabela 1 ).

Em relação à forma de pagamento do parto, $24(68,6 \%)$ mulheres entrevistadas procuraram o Sistema Ú nico de Saúde(SUS), sete (20\%) utilizaram planos privados de saúde e quatro $(11,4 \%)$ referiram sistema privado (Tabela 1$)$.

Foram encontradas associações entre a realização de cesariana e as categorias mais elevadas de classe econômica da ANEP e renda familiar per capita em salários mínimos. As razões deprevalência revelaram que as mulheres inseridas nas classes D e E tinham $58 \%$ menos probabilidade

Tabela 1. Descrição da amostra segundo algumas variáveis de assistência ao parto. São Leopoldo (RS), 2003.

\begin{tabular}{lr}
\hline \multicolumn{1}{c}{ Variável } & \multicolumn{1}{c}{ N $(\%)$} \\
\hline $\begin{array}{l}\text { Ganhou nenê nos últimos 12 meses } \\
\text { Sim }\end{array}$ & $36(3,5)$ \\
Não & $990(96,5)$ \\
Tipo de parto & \\
$\quad$ Normal & $17(47,2)$ \\
Cesárea & $19(52,8)$ \\
Quem fez o parto & \\
M édico & $35(97,2)$ \\
Ignorado & $1(2,8)$ \\
Pediatra em sala de parto & \\
Sim & $26(72,2)$ \\
Não & $8(22,2)$ \\
Ignorado & $2(5,6)$ \\
Financiamento do parto & \\
SU S & $24(68,6)$ \\
Planos privados de saúde & $7(20,0)$ \\
Particular & $4(11,4)$ \\
M unicípio da realização do parto & \\
São Leopoldo & $25(71,4)$ \\
N ovo Hamburgo & $8(22,9)$ \\
Porto Alegre & $1(2,9)$ \\
Sapucaia do Sul & $1(2,9)$ \\
\hline
\end{tabular}


de realização de cesariana quando comparadas com as classificadas nas classes A, B e C. De forma semelhante, as mulheres com renda familiar até um salário mínimo apresentaram prevalência de cesariana $63 \%$ menor do que as de maior renda (Tabela 2).

Em relação à forma definanciamento do parto, os dados revelaram quenenhuma mulher que recorreu ao sistema privado teve parto normal. A análise mostrou que as mulheres que consultaram no SU S tiveram $63 \%$ de proteção à ocorrência de cesárea em relação àquelas com planos privados de saúde e sistema privado (Tabela 2).

$N$ ão foram encontradas diferenças estatisticamentesignificativas entreo nível de escolaridade e o tipo de parto (Tabela 2).

A análise não mostrou diferenças estatisticamente significativas quanto à presença de pediatra no momento do parto e as variáveis socioeconômicas. Contudo, observou-se que as mulheres inseridas nas classes mais baixas e com menor renda tiveram um percentual menor depresença depediatra no momento do parto. Quanto à classe econômica, por exemplo, as mulheres inseridas nas classes $D$ eE apresentaram 2,5 vezesa possibilidade de não disporem da presença de pediatra para assistir ao recém-nascido quando comparadas com a categoria de base $\left(\mathrm{IC}_{95 \%} 0,59-10,62\right)$. Em relação à renda familiar, 33,3\% das mulheres com até um salário mínimo de renda per capita não tiveram a presença de pediatra no momento do parto (razão deprevalência: 4,33; IC ${ }_{95 \%} 0,60-31,32$ ).
Em relação ao financiamento do parto, os dados mostraram que nenhuma mulher classificada na categoria planos de saúde e sistema privado deixou de ter a presença de pediatra para assistir ao recém-nascido. Nesta análise, verificou-se que $30,4 \%$ das mulheres que receberam cuidados pelo SUS não manifestaram presença de pediatra no momento do parto.

\section{Discussão}

Entre as vantagens dos estudos de base populacional, destaca-se a possibilidade de se realizar inferências para toda a comunidade onde os dados foram coletados ${ }^{7}$. Este estudo incluiu de forma aleatória uma amostra representativa das mulheres residentes na cidade de São Leopoldo. A distribuição percentual das mulheresincluídas no estudo coincidiu com aquela verificada no Censo de 2000, realizado pelo IBGE².

Entre as limitações do estudo, deve-se destacar o baixo percentual de mulheres que tinham tido gestações nos doze meses que precederam as entrevistas, diminuindo o poder do estudo. 0 período de gestação escolhido para inclusão foi de doze meses, para se evitar problemas recordatórios. Contudo, estudos têm mostrado que as mães não esquecem o peso de nascimento de suas crianças ${ }^{8}$, fato que comprova a gestação e 0 parto como eventos vitais marcantes, de forma que não interfiram na lembrança.

Tabela 2. Análise bruta entre tipo de parto e variáveis socioeconômicas. São Leopoldo (RS), 2003.

\begin{tabular}{|c|c|c|c|c|c|}
\hline Variável & $\mathrm{N}$ & Cesariana (\%) & $\begin{array}{c}\text { Razão de } \\
\text { prevalência }\end{array}$ & $\begin{array}{l}\text { Intervalo de } \\
\text { confiança }_{95 \%}\end{array}$ & p-valor \\
\hline Classe econômica & & & & & 0,018 \\
\hline Classes A, B e C & 16 & $12(75,0)$ & 1,0 & & \\
\hline Classes D e E & 19 & $6(31,6)$ & 0,42 & $0,20-0,86$ & \\
\hline Renda familiar & & & & & 0,002 \\
\hline De 1,01 ou mais & 14 & $12(85,7)$ & 1,0 & & \\
\hline De 0 a 1 SM* & 22 & $7(31,8)$ & 0,37 & $0,19-0,71$ & \\
\hline Escolaridade & & & & & 0,065 \\
\hline 8 anos ou mais & 18 & $13(72,2)$ & 1,0 & & \\
\hline De 5 a 7 anos & 9 & $3(33,3)$ & 0,46 & $0,18-1,21$ & \\
\hline De 0 a 4 anos & 9 & $3(33,3)$ & 0,46 & $0,18-1,21$ & \\
\hline Financiamento do parto & & & & & 0,003 \\
\hline Planos de saúde e sistema privado & 11 & $10(90,9)$ & 1,0 & & \\
\hline SUS & 24 & $8(33,3)$ & 0,37 & $0,20-0,67$ & \\
\hline
\end{tabular}

*SM : salário mínimo. 
A prevalência de cesarianas encontradas neste estudo foi mais elevada do que as encontradas em outros estudos. Um estudo de coorte realizado em Pelotas (RS), durante o ano de 1993, mostrou uma prevalência de $30,5 \%{ }^{9}$. Estudo recente comparando o percentual de cesariana em Pelotas mostrou uma variação de 27,6\% em 1982 a $43,2 \%$ em 2004 , ou seja, um aumento de $55,6 \%{ }^{10}$. Os percentuais de parto por cesariana no Estado do Rio Grande do Sul entre 1995 e 2000 variaram de $33,4 \%$ a $25,5 \%$. No Brasil, também tem se observado uma ten dência na diminuição da prevalência acompanhando a queda de fecundidade; desta forma, em 1995, foi constatado que $32,4 \%$ foram cesarianas, enquanto que, em 2000 , o percentual foi de $23,8 \%{ }^{3}$.

Foram encontradas evidências mostrando a associação de tipo de parto e algumas variáveis que expressavam classe econômica. Da mesma forma, outros estudos também correlacionaram a ocorrência de parto por cesárea, segundo as condições sociais da gestante. Yazlle Rocha et al. ${ }^{11}$ estudaram retrospectivamente 36.956 casos de partos hospitalares na cidade de Ribeirão Preto (SP), ocorridos nos anos de 1978 a 1981, período no qual se instituiu no sistema previdenciário 0 mesmo pagamento, tanto para o parto por via vaginal quanto por cesárea. Confirmaram a existência deum gradiente de incidência de cesáreas, ou seja, ocorrência maior nas pacientes de me Ihor nível socioeconômico e menor naquelas de pior condição social. Esses autores afirmaram que a mudança na forma de pagamento da assistência ao parto não diminuiu a incidência de cesáreas e sugeriram que a diferença entre as modalidades assistenciais ao parto conduziu a diferentes padrões de assistência, interferindo na incidência de cesáreas. Barros et al..$^{12}$ estudaram a assistência pré-natal eao parto oferecido a mais de sete mil mulheres em Pelotas (RS), classificadas como sendo de baixo e alto risco e de alto e baixo ingresso, destacando a incidência de 50\% de cesáreas nas pacientes particulares e de $13 \%$ nas mulheres sem seguro saúde. Faúndes e $\mathrm{Ce}$ catti ${ }^{13}$ estudaram a proporção de cesáreas entre os partos assistidos pela Previdência Social no Brasil, de 1970 a 1980. Relataram que a incidência era maior nas capitais do que nas cidades do interior, nas macrorregiões mais ricas do país e entre as famílias de renda mais elevada e consideraram que os fatores socioculturais, institucionais, legais e a organização da assistência obstétrica poderiam interferir nesse processo.

Diversos estudos, principalmente na área materno-infantil, têm revelado indesejáveis ini- quidades na distribuição da assistência à saúde no Brasil ${ }^{14}$. Essas iniquidades sempre mostraram que as pessoas com maior necessidade de cuidados invariavelmentereceberam menor atenção. Contudo, no caso do tipo de parto, em São Leopoldo e no Brasil, esta lógica está invertida, uma vez que o parto cesáreo tem maior probabilidade deproduzir efeitos adversos do que o de tipo normal.

Em estudo realizado na cidade de Ribeirão Preto no período entre 1986 e 1995, a distribuição dos partos, segundo a categoria de admissão, correspondeu a 5,4\% na categoria privada, $28,7 \%$ na de pré-pagamento e $65,9 \%$ na categoria SUS, sendo a distribuição entre parto normal e cesárea muito semelhante, 48,8\% e 48,3\%, respectivamente. Ainda neste estudo, em 1995, foram encontrados os seguintes percentuais de cesáreas conforme as categorias de financiamento: $81,8 \%$ no sistema privado, $77,9 \%$ no sistema de pré-pagamento e $32,1 \%$ no SUS ${ }^{15}$.

Deve-se ressaltar que, para possibilitar a análise estatística, as variáveis foram agrupadas de tal forma que as categorias nas quais foram encontradas associações traduzem condições socioeconômicas extremamente desfavoráveis, o que pode ser uma confirmação de uma iniquidade indesejável do ponto de vista de adequada prestação de assistência à saúde.

Contribuindo para a discussão deste estudo, Gentile et al. ${ }^{16}$ compararam os partos ocorridos em nove maternidades privadas do Rio de Janeiro, entre 1968 e 1993, e verificaram que houve aumento significativo de cesáreas, apesar de terem sido igualados os honorários médicos para o parto por cesárea e por via vaginal. Sugerem os citados autores que não é a forma de remuneração da assistência ao parto, isoladamente, que interfereno índice de cesáreas, mas sim uma série devariáveisquepodem influenciar no tipo decontrato quese estabeleceentrea pacientee o médico.

Contudo, sabe-se que a remuneração para os profissionais por tipo de parto no Sistema Ú nico de Saúde foi equiparada, mas esta relação não está mantida nos planos privados de saúde, nos quais o valor da cesariana é superior ao do parto normal.

O paradoxo observado de incidência maior de cesáreas nos grupos de menor risco e com melhor nível de saúde estimulou a procura de explicações extratécnicas. Yazlle Rocha et al. ${ }^{11}$, Barros et al. ${ }^{12}$ e Gentile et al. ${ }^{16}$ trataram de encontrar explicações nos padrões assistenciais diferenciados e no tipo de relação que se estabelece entre o médico e a paciente e os seus familiares. 
Assim, se for assumida a indicação por fatores extratécnicos, tais como padrão assistencial e contrato, poderia ser explicada a el evação dessas taxas, pois responderia o desejo da paciente e/ou da família de um parto com resolução "segura" e sem sofrimento, como é culturalmente difundido no Brasil. N esse caso, a cesárea, como modalidade de resolução ao parto, equivale a um recurso técnico a ser incorporado por quem o deseja e tem poder de custeá-lo. Esse tipo de parto teria sido transformado em objeto de consumo acessível segundo o padrão de renda, o que viria explicar ser mais incidente nos grupos de maior renda, embora de menor risco obstétrico ${ }^{15}$.

Talvez a maior contribuição do presente estudo foi verificar a fonte financiadora do parto. Assim, foi possível afirmar que a remuneração interferiu no tipo departo. Contudo, deve-sealertar que o percentual de cesáreas nas mulheres que recorreram ao Sistema Ú nico de Saúde também foi elevado, atingindo 33,3\%. Desta forma, sugere-se que outros estudos da mesma natureza aprofundem esta questão, agregando, por

\section{Referências}

1. Menezes AMB, Hallal PC, Santos IS, Victora CG Barros FC. Infant mortality in Pelotas, Brazil: a comparison of risk factors in two birth cohorts. Rev Panam Salud Publica 2005; 18(6):439-446.

2. Olinto MTA, Dias da Costa JS, Mendes KG. A Saúde em São Leopoldo - Relatório 2000. São Leopoldo: Editora UNISIN OS; 2003.

3. Brasil. Ministério da Saúde. Assistência à saúde no SUS: média e alta complexidade - 1995/2000. Brasília: Ministério da Saúde; 2001.

4. Belizán JM, Althabe F, Barros FC, Alexander S. Rates and implications of caeserean sections in Latin America: ecological study. Br M ed J 1999; 319:1397-1402.

5. Rezende J. O peração cesariana. In: Rezende J, organizador. Obstetrícia. $8^{a}$ ed. Rio de Janeiro: Guanabara-Koogan; 1998. p. 1173-1232.

6. Beaglehole R, Bonita R, Kjellström T. Epidemiologia básica. São Paulo: Santos; 2001.

7. Andrade ALSS, Zicker F. Estudos de prevalência. In: Andrade ALSS, Zicker F, organizadores. M étodos de investigação epidemiológica em doenças transmissíveis. Brasília: Organização Pan-Americana da Saúde/Fundação Nacional de Saúde; 1997. p. 33-42.

8. Victora CG, Barros FC, M artines JC, Béria JU, Vaughan JP. As mães lembram o peso ao nascer de seus filhos? Rev. Saude Publica 1985; 3(19):195-200.

9. Dias da Costa JS, Victora CG, Barros FC, Halpern R, Horta BL, Manzolli P. Assistência médica maternoinfantil em duas coortes de base populacional no Sul do Brasil: tendências e diferenciais. Cad Saude Publica 1996; 12(Sup1.1):59-66. exemplo, o tempo despendido pelos profissionais como uma medida indireta de custo.

A pesar da ausência de associação estatística, provavelmente relacionada à falta de poder do estudo na análise da presença de pediatra no momento do parto, deve-se ressaltar que os resultados mostraram um percentual elevado de partos sem a presença de pediatra para avaliar 0 recém-nascido, evidenciando outra indesejável iniquidade na assistência de São Leopoldo.

\section{Colaboradores}

M Schreiner participou da coleta e análise dos dados e da redação do artigo. JSD da Costa participou da elaboração do projeto de pesquisa, da análise dos dados e da redação do artigo. M TA Olinto eS M eneghel participaram da elaboração do projeto de pesquisa, da coleta e análise dos dados e da redação do artigo.

10. Barros FC, Victora CG, Barros AJD, Santos IS, Albernaz EP, M atijasevich A, Domingues M R, Sclowitz IKT, $H$ allal PC, Silveira MF, Vaughan JP. The challenge of reducing neonatal mortality in middle-income countries: findings from three Brazilian birth cohorts in 1982, 1993 and 2004. Lancet 2005; 365:847-854.

11. Yazlle Rocha JS, Ortiz PC, Fung YT. A incidência de cesáreas e a remuneração da assistência ao parto. Cad Saude Publica 1985; 1:457-466.

12. Barros FC, Vaugham JP, Victora CG. Why so many caesarean sections? The need for a further policy change in Brazil. Health Policy Plan 1986; 1:19-29.

13. Faúndes A, Cecatti JG. A operação cesárea no Brasil: incidência, tendências, causas, consequencia e propostas de ação. Cad Saude Publica 1991; 7:150-173.

14. Victora CG, Vaughan JP, Barros F, Silva AC, Tomasi E. Explaining trends in inequities: evidence from Brazilian child health studies. Lancet 2000; 356:1093-1098.

15. Yazlle MEHD, Yazlle Rocha JS, M endes MC, Patta MC, M arcolin AC, Azevedo GD. Incidência de cesáreas segundo fonte de financiamento da assistência ao parto. Rev. Saude Publica 2001; 35(2):202-206.

16. Gentile FP, Noronha Filho G, Cunha AA. Associação entre a remuneração da assistência ao parto e a prevalência de cesariana em maternidades do Rio de Janeiro: uma revisão da hipótese de Carlos Gentile de Mello. Cad Saude Publica 1997; 13:221-226.

Artigo apresentado em 12/06/2007

Aprovado em 14/12/2007 